\title{
Does bicompartmental knee arthroplasty hold an advantage over total knee arthroplasty? Systematic review and meta-analysis
}

\author{
Hany Elbardesy ${ }^{1}{ }^{*}$, Ahmed K. Awad ${ }^{2}$, André McLeod ${ }^{1}$, Samar Tarek Farahat ${ }^{3}$, \\ Somaya Zain Elabdeen Sayed ${ }^{4}$, Shane Guerin ${ }^{1}$, and James Harty ${ }^{1}$ \\ 1 Department of Trauma and Orthopaedic, Cork University Hospital, Wilton, Cork T12DFK4, Ireland \\ ${ }^{2}$ School of Medicine, Ain-Shams University, Cairo 11566, Egypt \\ 3 Faculty of Medicine, Menofia University, Menofia 51132, Egypt \\ ${ }^{4}$ Faculty of Medicine, Minia University, Minia 61519, Egypt
}

Received 29 April 2021, Accepted 12 June 2021, Published online 9 July 2021

\begin{abstract}
Introduction: The role of bicompartmental knee arthroplasty (BKA) in the treatment of medial patellofemoral osteoarthritis (MPFOA) has been debated by orthopaedic surgeons for years. The BKA is a cruciate ligament retaining prosthesis designed to mimic the kinematics of the native knee that requires resurfacing of only two knee compartments. In this study, we aim to assess the patient recorded outcome measures (PROMs), range of motion (ROM), perioperative morbidity, and implant revision rate in patients undergoing BKA and compare them to those undergoing total knee arthroplasty (TKA) for bicompartmental knee osteoarthritis (OA). Patients and methods: We followed the Preferred Reporting Items for Systematic Reviews and Meta-analyses Statement (PRISMA). Articles from any country and written in any language were considered. We included all randomized control trials and retrospective cohort studies examining BKA versus TKA for bicompartmental knee OA. The primary outcome measure was knee society score (KSS) at one year and the secondary outcome measures were Oxford knee score (OKS) and short-form survey (SF-)12 at six and twelve months. Results: We included five studies in our meta-analysis. In terms of OKS, KSS, and SF-12, our meta-analysis suggests better short-term results for the TKA compared with the BKA. TKA was also associated with a shorter operative time and a lower revision rate. The BKA implant did however result in marginally less intraoperative blood loss and slightly better post-operative ROM. Conclusions: BKA did not prove to be an equivalent alternative to TKA in bicompartmental knee OA. It was associated with inferior KSS, OKS, and SF-12 at short-term follow-up and a higher revision rate.
\end{abstract}

Key words: Uni knee, Patellofemoral arthroplasty, Total knee arthroplasty, Meta-analysis.

\section{Introduction}

Total knee arthroplasty (TKA) is a recognized treatment option for knee osteoarthritis representing $84.3 \%$ of the total number of knee arthroplasty reported by the Australian Orthopaedic Association National Joint Replacement Registry (AOANJRR) over the last 15 years. This is in comparison to partial knee arthroplasty which accounts for only $7.7 \%$ of this cohort [1]. The recorded survival rate at 25 years follow-up was $82 \%$ for TKAs compared with $70 \%$ for Uni-compartmental Knee Arthroplasty (UKA) [2]. A recent study showed that of the patients waiting for knee arthroplasty, $51 \%$ had medial compartment OA, $6.5 \%$ had lateral compartment OA, and $1.2 \%$ had patellofemoral osteoarthritis (PFOA). Tri-compartmental OA was found only in $16.7 \%$ and medial patellofemoral osteoarthritis

\footnotetext{
*Corresponding author: elbardecy@hotmail.com
}

(MPFOA), a combination of the medial compartment and patellofemoral OA, was found in $15.5 \%$ [3]. This suggests that a proportion of patients who receive TKAs are undergoing resurfacing of a non-arthritic lateral compartment with intact anterior cruciate ligament (ACL). In theory, these patients could be adequately managed with bicompartmental knee arthroplasty (BKA) resulting in reduced intra-operative blood loss and preserving the cruciate ligaments thus maintaining the natural kinematics of the native knee $[4,5]$. Some authors have advocated for bi-compartmental knee replacement as it is associated with less blood loss, fewer side effects, and quicker rehabilitation than TKA [6]. As such, selective knee compartment replacement has been adopted by some researchers with promising outcomes [7, 8]. Bi-Compartmental OA (BCOA) was not traditionally recommended for UKA. Thus, BKA was advocated to be used in the treatment of BCOA [3, 7]. This meta-analysis aims to evaluate studies assessing Patient 


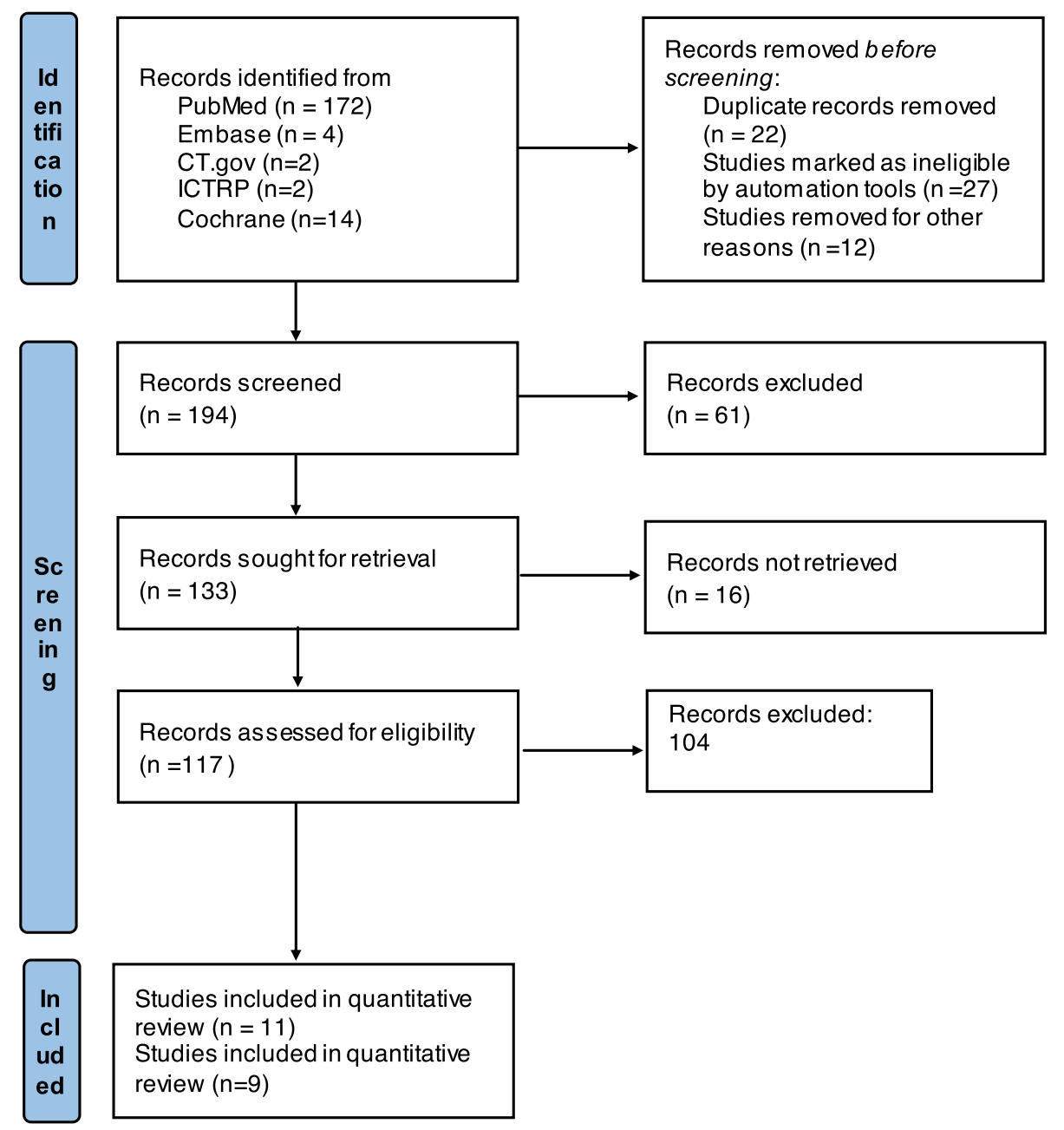

Figure 1. Preferred reporting items for systematic reviews and meta-analyses (PRISMA) flow chart.

Recorded Outcome Measures (PROMs) for both BKA and TKA for the treatment of BCOA and to use a systematic approach to comparatively evaluate variables including operating time, postoperative Range of Motion (ROM), intraoperative blood loss, and revision rate.

\section{Materials and methods}

We followed in this review both the Preferred Reporting Items for Systematic Reviews and Meta-analyses Statement (PRISMA), (Fig. 1), and the Cochrane Handbook for systematic reviews and meta-analysis [9]. We conducted an initial search using Web of Science, PubMed, EMBASE-OVID, Google Scholar, and Cochrane Library. We used the following keywords and their combinations: medial pivot, posterior stabilized, and total knee arthroplasty. Articles published up to March 2021 were included in our literature search and were limited to studies in human subjects published in any language. Additionally, we cross-referenced the bibliographies of retrieved articles and review papers to ensure that we captured all relevant studies.

\section{Study selection criteria}

We included all comparative studies (retrospective/ prospective cohorts, randomized clinical trials (RCTs)) involving patients undergoing unilateral or bilateral TKAs which were of MP or PS design, and where outcomes were compared between the two designs. We excluded cadaveric, in vitro, or single-arm studies. Conference abstracts, letters to the editor, reviews were also excluded.

\section{Data extraction and analysis}

Five authors independently screened all titles and abstracts identified by the initial search to assess their eligibility for inclusion. We then did a full screening of each manuscript and conducted a final assessment of the eligibility for all included studies. The same reviewers performed the data extraction. Any discrepancies found after data collection were resolved by discussion between all reviewers. The collected information included first author, year, journal, country, level of evidence, study design, number of centers, study length, number of participants, age, gender, and Body Mass Index (BMI). 


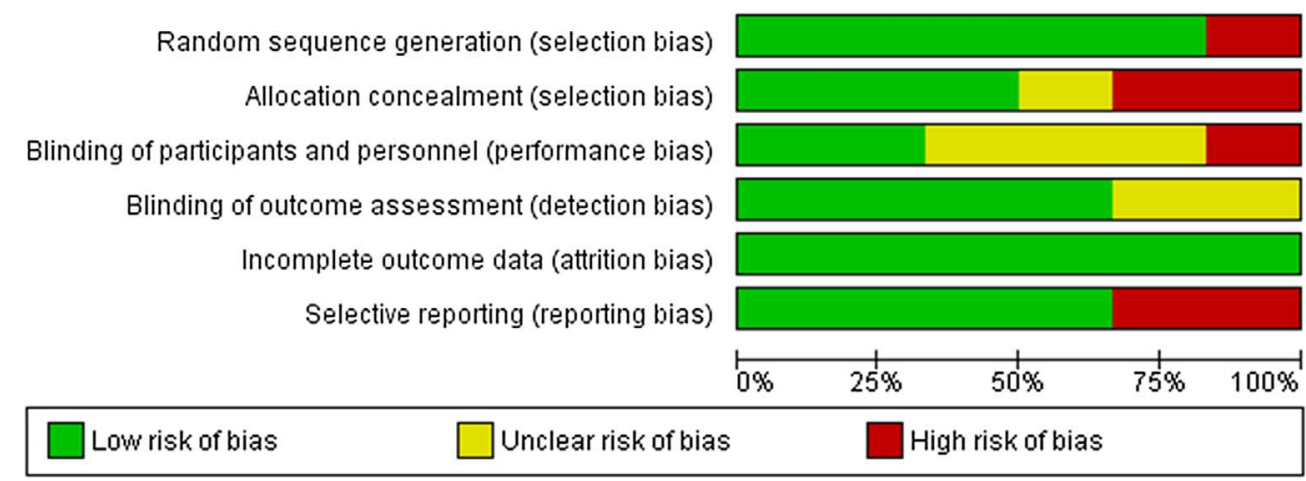

Figure 2. Risk of bias graph: review authors' judgments about each risk of bias item presented as percentages across all included studies.

\section{Methodological quality assessment}

We assessed the risk of bias for RCTs by using the Cochrane risk of bias criteria and the nonrandomized cohort studies using the Newcastle-Ottawa Scale [9, 10]. Five reviewers independently crossed-checked the quality of the included studies. Disagreements were resolved through discussion.

\section{Risk of Bias (ROB) assessment}

Six RCTs were assessed for potential bias using the Cochrane risk of bias tool. A summary of the risk of bias is shown in (Figs. 2 and 3). Randomization and patient blinding were adequate in five studies [11-15], and unclear in the other one study [16]. A high risk of bias was not found in any of the six studies, yet some concerns were raised regarding one of the domains of all six studies. All the studies had clear judgment in at least one of the domains. Quality assessment of five nonrandomized cohort studies using the Newcastle-Ottawa Scale showed that all studies were of high quality (Table 1).

\section{Outcome measures}

We assessed three variables in our meta-analysis, Oxford Knee Score (OKS) [17], Knee Society Score (KSS) [18], and 12-Item Short Form Survey (SF-12) [19]. We examined four other variables in our systematic review including operating time, postoperative Range of Motion (ROM), intraoperative blood loss, and revision rate. Sufficient data were not available to conduct a meta-analysis on these variables.

\section{Statistical analysis}

We conducted a statistical analysis by using Review Manager (RevMan), version 5.3 (The Nordic Cochrane Centre, The Cochrane Collaboration, 2009, Copenhagen, Denmark) [20]. Heterogeneity between studies was assessed by the $I^{2}$ statistic and a $c^{2}$ of $<0.05$ was used to define the significance of the heterogeneity among the included studies. Ranges of $0-24 \%, 25-74 \%$, and $75-100 \%$ were used to define minor, moderate, and major heterogeneity respectively [9]. Mean

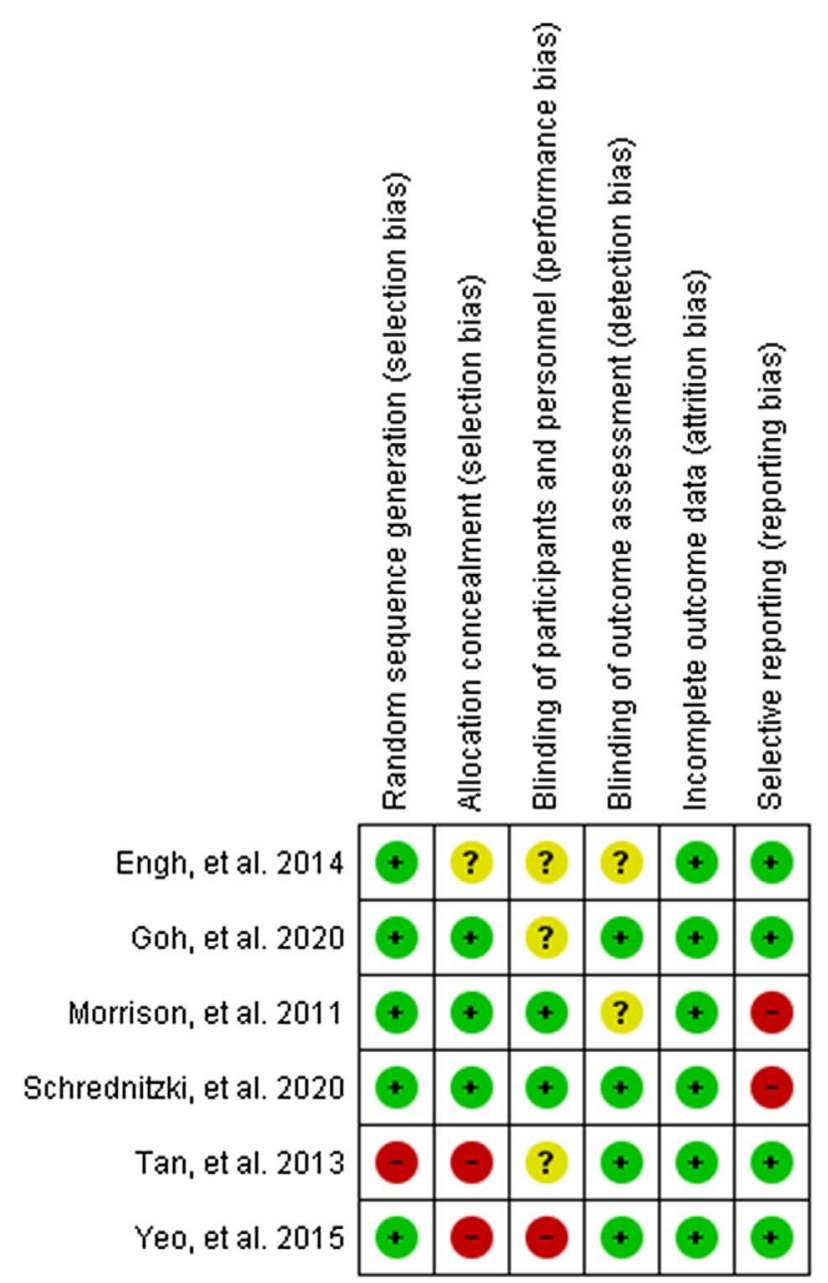

Figure 3. Risk of bias summary: review authors' judgments about each risk of bias item for each included study.

differences and standard deviations (SDs) were used for continuous variables. We used the random-effects model in our meta-analysis. We illustrated the results using forest plots, which used a $95 \%$ confidence interval (CI) for each study and a cumulative weighted Mean Difference (MD) for all the included studies [9]. 
Table 1. Newcastle-Ottawa Scale (NOS) for assessing the quality of observational studies.

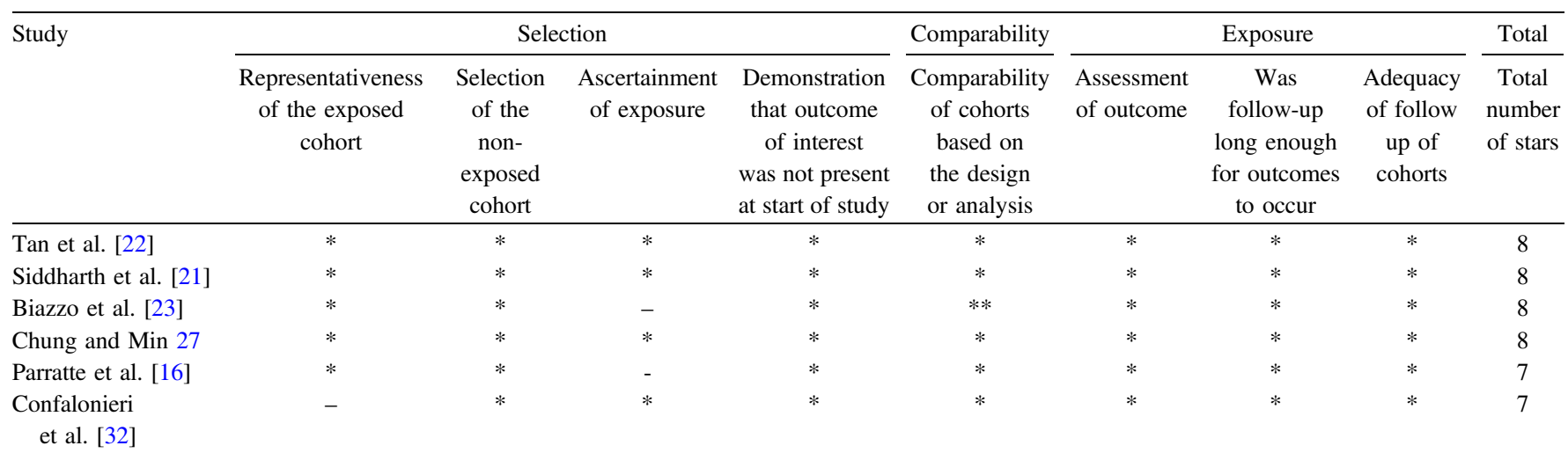

\section{Results}

\section{Study characteristics}

Our literature review returned 172 articles after the removal of duplicates. Title and abstract screening revealed 133 articles that were eligible for full-text screening. 121 articles were subsequently excluded for not meeting selection criteria leaving eleven articles that were included for qualitative review. Nine of these articles were included in the meta-analysis. A flow chart demonstrating the study selection process is provided (Fig. 1). Six studies were randomized clinical trials (RCTs), and five were retrospective cohort studies. A summary of the characteristics of included studies is provided (Table 2).

\section{Patient baseline characteristics}

Our review included 561 knees (310 in BKA group, 251 in TKA group). The BKA group had an average age of 59.25 years $( \pm 6.25$ years), of which 183 out of 310 patients $(60.65 \%)$ were female, with an average body mass index of $28.37 \mathrm{~kg} / \mathrm{m}^{2}( \pm 3.32)$. The TKA cohort had a patient distribution with an average age of 62.69 years ( \pm 5.5 years), of which $167 /$ $251(66.53 \%)$ were female, with an average body mass index of $29.3 \mathrm{~kg} / \mathrm{m}^{2}( \pm 3.76)$. A summary of the patient demographics of included studies is provided (Table 3 ).

\section{Meta-analysis}

Our meta-analysis comparatively assessed the KSS, OKS, and SF-12 scores at six months and one-year follow-up.

\section{Oxford knee score}

Overall, four studies including 288 knees (167 BKA; 121 TKA) reported on OKS after six months and one year. They reported a significantly higher OKS for the TKA cohort at six months and marginal improvement at one-year follow-up. Three studies reported on OKS after 5 years encompassing 219 knees (112 BKA and 107 TKA). They reported a significantly superior OKS for the TKA cohort. Heterogeneity analysis demonstrated high statistical evidence for variation within the studies $\left(I^{2}=93 \%\right)$. The cumulative MD was significant at $-3.43(95 \% \mathrm{CI},-5.0-1.86 ; P<0.001)$ (Fig. 4).

\section{Knee society score}

Overall, seven studies including 459 knees reported on postoperative KSS score after one year. They reported a significantly better KSS for the TKA cohort. Heterogeneity analysis demonstrated high statistical evidence for heterogeneity $\left(I^{2}=97 \%\right)$. The cumulative MD was significant at -3.43 (95\% CI, $-5.70-1.16 ; P<0.005$, Fig. 5).

\section{SF-12 score}

Four studies including 292 knees (169 BKA; 123TKA) reported on SF-12 after six months and one year. They reported significantly higher SF-12 scores for the TKA cohort at six and twelve months. The heterogeneity analysis demonstrated no statistical evidence for variation within the study $\left(I^{2}=0 \%\right)$. The cumulative MD was significant at -1.49 (95\% CI, $-2.31-0.07 ; P<0.001$ ) (Fig. 6).

\section{Systematic review}

\section{ROM}

ROM was marginally greater in the BCA cohort $[13,16$, $21,22]$. One study reported an improvement in the degree of knee flexion [16].

\section{Operative time}

The mean time for surgery was significantly longer for BCA $(73.5 \pm 9.9 \mathrm{~min})$ compared to TKA $(58.8 \pm 12.8 \mathrm{~min}$; $P<0.05)[13,22,23]$.

\section{Blood loss}

A higher average blood loss was reported in the BCA group in only one study [13]. It was reported as being significantly less than the TKA group in four studies $(P<0.05)$ [12, 15, 22, 23]. 
Table 2. Study characteristics.

\begin{tabular}{|c|c|c|c|c|c|c|}
\hline Study & Year & Country & Journal & $\begin{array}{l}\text { Study } \\
\text { type }\end{array}$ & PROM & Revision Rate \\
\hline $\begin{array}{l}\text { Schrednitzki } \\
\text { et al. [13] }\end{array}$ & 2020 & Germany & The journal of arthroplasty & RCT & $\begin{array}{l}\text { KSS, OKS, and the } \\
\text { University of California, } \\
\text { Los Angeles scores, SF-12 }\end{array}$ & 1 at BKA group \\
\hline Yeo et al. [12] & 2015 & Singapore & The knee & RCT & $\begin{array}{l}\text { BKS, OKS, AKSS, pre and } \\
\text { post-operative range of } \\
\text { motionSF-12 }\end{array}$ & 1 at BKA group \\
\hline Tan et al. [22] & 2013 & Singapore & $\begin{array}{l}\text { Journal of Orthopaedic } \\
\quad \text { Surgery }\end{array}$ & $\mathrm{RCT}$ & $\begin{array}{l}\text { KSS, WOMAC, and SF-36 } \\
\text { scores, range of movement, } \\
\text { Pain score (VAS) }\end{array}$ & 0 at both groups \\
\hline $\begin{array}{l}\text { Siddharth } \\
\quad \text { et al. [21] }\end{array}$ & 2013 & Singapore & The journal of knee surgery & RCS & $\begin{array}{l}\text { KSS-clinical, KSS-function, } \\
\text { KSS-total, KOOS-pain, } \\
\text { KOOS symptoms,KOOS- } \\
\text { stiffness, and KOOS-ADL } \\
\text { WOMAC pain }\end{array}$ & 1 at BKA group \\
\hline Morrison et al. [11] & 2011 & USA & The journal of arthroplasty & RCT & SF-12 and WOMAC & 3 at BKA group \\
\hline Engh et al. [14] & 2014 & USA & The journal of arthroplasty & $\mathrm{RCT}$ & KSS, OKS & $\begin{array}{l}3 \text { at BKA group, } \\
\text { and } 1 \text { at TKA group }\end{array}$ \\
\hline Biazzo et al. [23] & 2019 & Italy & $\begin{array}{l}\text { MUSCULOSKELETAL } \\
\text { SURGERY }\end{array}$ & RCS & KSS & 1 at TKA group \\
\hline $\begin{array}{l}\text { Chung and } \\
\text { Min } 27\end{array}$ & 2013 & Korea & KSSTA & PCS & $\begin{array}{l}\text { knee extensor and flexor } \\
\text { torque, hamstring/ } \\
\text { Quadriceps }(\mathrm{H} / \mathrm{Q}) \text { ratio, } \\
\text { knee position sense, and } \\
\text { physical performance, } \\
\text { proprioception }\end{array}$ & N/A \\
\hline $\begin{array}{l}\text { Parratte } \\
\quad \text { et al. [16] }\end{array}$ & 2015 & $\begin{array}{l}\text { Belgium } \\
\text { and France }\end{array}$ & $\begin{array}{l}\text { Orthopaedics \& Traumatology: } \\
\text { Surgery \& Research }\end{array}$ & RCS & $\begin{array}{l}\text { KSS function, KSS knee, } \\
\text { UCLA score. }\end{array}$ & N/A \\
\hline $\begin{array}{r}\text { Confalonieri } \\
\text { et al. [32] }\end{array}$ & 2009 & Italy & Arch Orthop Trauma Surg & RCS & $\begin{array}{l}\text { Post-op HKA angle, IKS } \\
\text { score, FUNCT score, } \\
\text { GIUM scoreWOMAC } \\
\text { pain, function, and } \\
\text { stiffness }\end{array}$ & N/A \\
\hline Goh et al. [15] & 2020 & Singapore & The Knee & RCT & $\begin{array}{l}\text { KSS function, KSS knee, } \\
\text { OKS, SF36PCS, SF36 } \\
\text { MCS, knee flexion }\end{array}$ & 1 TKA group \\
\hline
\end{tabular}

Note: KSS: Knee society score, OKS: Oxford knee score, AKSS: American knee society score, SF-12: Short Form 12, WOMAC: Western Ontario and McMaster Universities Osteoarthritis Index assessments, RCT: randomized control study, RCS: retrospective cohort study, PCS prospective cohort study, KSSTA: Knee Surg Sports Traumatology Arthroscopy.

Table 3. Patient's demographics.

\begin{tabular}{|c|c|c|c|c|c|c|c|c|c|c|c|c|}
\hline \multirow[t]{2}{*}{ Study } & \multirow[t]{2}{*}{ Centers } & \multirow{2}{*}{$\begin{array}{c}\text { Total } \\
\text { number }\end{array}$} & \multirow[t]{2}{*}{ BKA } & \multirow[t]{2}{*}{ TKA } & \multicolumn{2}{|c|}{$\begin{array}{l}\text { Gender } \\
\text { female }\end{array}$} & \multicolumn{2}{|c|}{ Age (SD) } & \multicolumn{2}{|c|}{ BMI (SD) } & \multicolumn{2}{|c|}{ Follow-up } \\
\hline & & & & & BKA & TKA & BKA & TKA & BKA & TKA & BKA & TKA \\
\hline Schrednitzki et al. [13] & one center & 80 & 40 & 40 & 20 & 20 & $65.25(8.9)$ & $63.55(6.6)$ & $32.9(6.1)$ & $34.7(6.5)$ & 5 years & 5 years \\
\hline Yeo et al. [12] & one center & 48 & 26 & 22 & 21 & 16 & $63.8(8.03)$ & $63.1(7.3)$ & $27.28(3.04)$ & $28.15(4.52)$ & 5 years & 5 years \\
\hline Tan et al. [22] & One center & 27 & 15 & 12 & 8 & 9 & $52(41-62)$ & $60(41-63)$ & $26.0(4.2)$ & $28.3(4.9)$ & 20 months & 20 months \\
\hline Siddharth et al. [21] & one center & 36 & 16 & 20 & 10 & 16 & $52.1(6.4)$ & $65.1(7)$ & $27.6(4.4)$ & $27.3(3.8)$ & 2 years & 2 years \\
\hline Morrison et al. [11] & one center & 71 & 50 & 21 & 25 & 15 & $63.2(11.5)$ & $67.18(9.5)$ & $31.7(7.7)$ & $33.7(8.6)$ & 2 years & 2 years \\
\hline Engh et al. [14] & One center & 75 & 50 & 25 & 25 & 12 & 60.3 & 58.3 & 28.8 & 30 & 2 years & 2 years \\
\hline Biazzo et al. [23] & One center & 40 & 20 & 20 & 16 & 17 & 67 & 65 & 27.6 & 29.7 & 38 months & 38 months \\
\hline Chung and Min 27 & One center & 24 & 11 & 13 & 7 & 11 & $54.8(5.6)$ & $65.7(6.7)$ & $27(2.8)$ & $25.4(2.5)$ & 12 months & 12 months \\
\hline Parratte et al. [16] & Two center & 68 & 34 & 34 & 21 & 21 & $61(7)$ & $61(8)$ & $27.5(4)$ & $27.5(4.5)$ & 2 years & 2 years \\
\hline Confalonieri et al. [32] & One center & 44 & 22 & 22 & 14 & 14 & $60.4(6.06)$ & $60.7(5.96)$ & N/A & N/A & 48 months & 48 months \\
\hline Goh et al. [15] & One center & 48 & 26 & 22 & 21 & 16 & $63.8(8.03)$ & $63.1(7.34)$ & $27.28(3.04)$ & $28.15(4.52)$ & 10 years & 10 years \\
\hline
\end{tabular}

SD: standard deviation. 


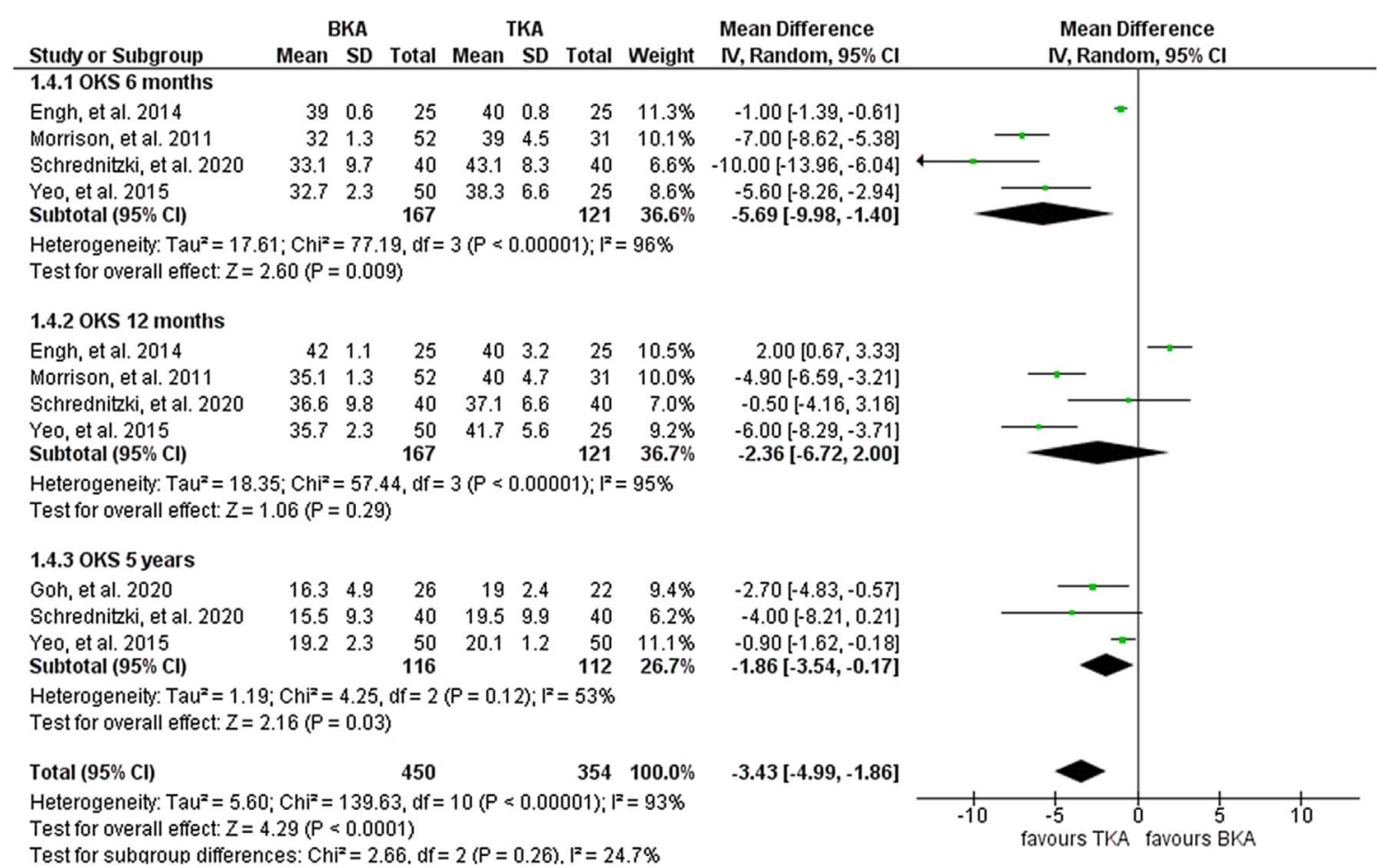

Figure 4. Forest plot of comparison: BKA vs TKA, outcome: OKS after 6, 12 months, and 5 years.

\begin{tabular}{|c|c|c|c|c|c|c|c|c|c|c|c|c|}
\hline \multirow{3}{*}{$\begin{array}{l}\text { Study or Subgroup } \\
1.7 .2 \text { KSS } 12 \text { months }\end{array}$} & \multicolumn{3}{|c|}{ BKA } & \multicolumn{3}{|c|}{ TKA } & \multirow{3}{*}{\multicolumn{2}{|c|}{$\begin{array}{cl} & \text { Mean Difference } \\
\text { Weight } & \mathrm{IV}, \text { Random, } 95 \% \mathrm{Cl} \\
\end{array}$}} & \multirow{2}{*}{\multicolumn{4}{|c|}{$\begin{array}{c}\text { Mean Difference } \\
\text { N, Random, } 95 \% \mathrm{Cl}\end{array}$}} \\
\hline & Mean & SD & Total & Mean & SD & Total & & & & & & \\
\hline & & & & & & & & & & & & \\
\hline Biazzo, et al. 2018 & 92.5 & 2 & 40 & 94.3 & 2 & 40 & $17.0 \%$ & $-1.80[-2.68,-0.92]$ & & $\rightarrow$ & & \\
\hline Engh, et al. 2014 & 90.5 & 1.2 & 25 & 92.7 & 2.1 & 25 & $17.0 \%$ & $-2.20[-3.15,-1.25]$ & & $\rightarrow$ & & \\
\hline Parratte, et al. 2015 & 82 & 0.1 & 34 & 88.7 & 1.5 & 34 & $17.3 \%$ & $-6.70[-7.21,-6.19]$ & & $=$ & & \\
\hline Schrednitzki, et al. 2020 & 88.5 & 12.2 & 40 & 92.4 & 8.9 & 40 & $10.0 \%$ & $-3.90[-8.58,0.78]$ & & & & \\
\hline Siddharth, et al. 2013 & 90.5 & 7.9 & 26 & 92.6 & 8.8 & 26 & $10.3 \%$ & $-2.10[-6.65,2.45]$ & & & & \\
\hline Tan, et al. 2013 & 84 & 9.5 & 27 & 90 & 5.5 & 27 & $11.1 \%$ & $-6.00[-10.14,-1.86]$ & & & & \\
\hline $\begin{array}{l}\text { Yeo, et al. } 2015 \\
\text { Subtotal }(95 \% \mathrm{Cl})\end{array}$ & 90.7 & 1.2 & $\begin{array}{r}50 \\
242\end{array}$ & 92.56 & 1.1 & $\begin{array}{r}25 \\
217\end{array}$ & $\begin{array}{r}17.3 \% \\
100.0 \%\end{array}$ & $\begin{array}{l}-1.86[-2.40,-1.32] \\
-3.43[-5.70,-1.16]\end{array}$ & & & & \\
\hline \multicolumn{13}{|c|}{$\begin{array}{l}\text { Heterogeneity: Tau }=7.67 ; \mathrm{Chi}^{2}=207.75, \mathrm{df}=6(\mathrm{P}<0.00001) ; \mathrm{l}^{2}=97 \% \\
\text { Test for overall effect: } Z=2.96(\mathrm{P}=0.003)\end{array}$} \\
\hline Total $(95 \% \mathrm{Cl})$ & & & 242 & & & 217 & $100.0 \%$ & $-3.43[-5.70,-1.16]$ & & & & \\
\hline \multicolumn{9}{|c|}{$\begin{array}{l}\text { Heterogeneity: } \mathrm{Tau}^{2}=7.67 ; \mathrm{Chi}^{2}=207.75, \mathrm{df}=6(P<0.00001) ; \mathrm{I}^{2}=97 \% \\
\text { Test for overall effect: } Z=2.96(P=0.003) \\
\text { Test for subqroup differences: Not applicable }\end{array}$} & -10 & $\begin{array}{c}-5 \\
\text { Favours TKA }\end{array}$ & $\begin{array}{cc}5 \\
\text { Favours } B K A\end{array}$ & 10 \\
\hline
\end{tabular}

Figure 5. Forest plot of comparison: BKA vs TKA, outcome: KSS after 12 months.

\section{Revision rate}

About the postoperative revision rate, the BKA cohort reported 9 cases out of $310(2.9 \%)$ after a mean follow-up of 45 months. However, the TKA reported only 3 revisions out of $251(1.2 \%)$ within the same time frame.

\section{Discussion}

The most important findings in this meta-analysis were that the post-operative KSS, OKS, and SF-12 were significantly higher in the TKA cohort compared with the BKA. However, the BKA showed less intraoperative bleeding and a slight but insignificant superiority in terms of post-operative ROM.
To our knowledge, only one meta-analysis (MA) [24], has been published examining the same topic with the inclusion of seven studies in their qualitative review and two studies in the MA. The included studies in that article were published up to September 2015. Five studies examining this topic have been published since that date and are all included in our study. This may explain the contradiction in the results between the two articles. The survivorship of BKAs is debatable. One author reported $80 \%$ survivorship of BKA at 17 years [25], while another study including only nine patients showed $100 \%$ survival of the BKA at 12 years with very good functional outcomes [26]. Parratte et al. [25], examined 71 patients with BKAs and reported a 54\% survival rate at 17 years to follow up. This is more than five times the revision rate reported for the TKA in another study which was $9.0 \%$ at 19 years [1]. Of note, 


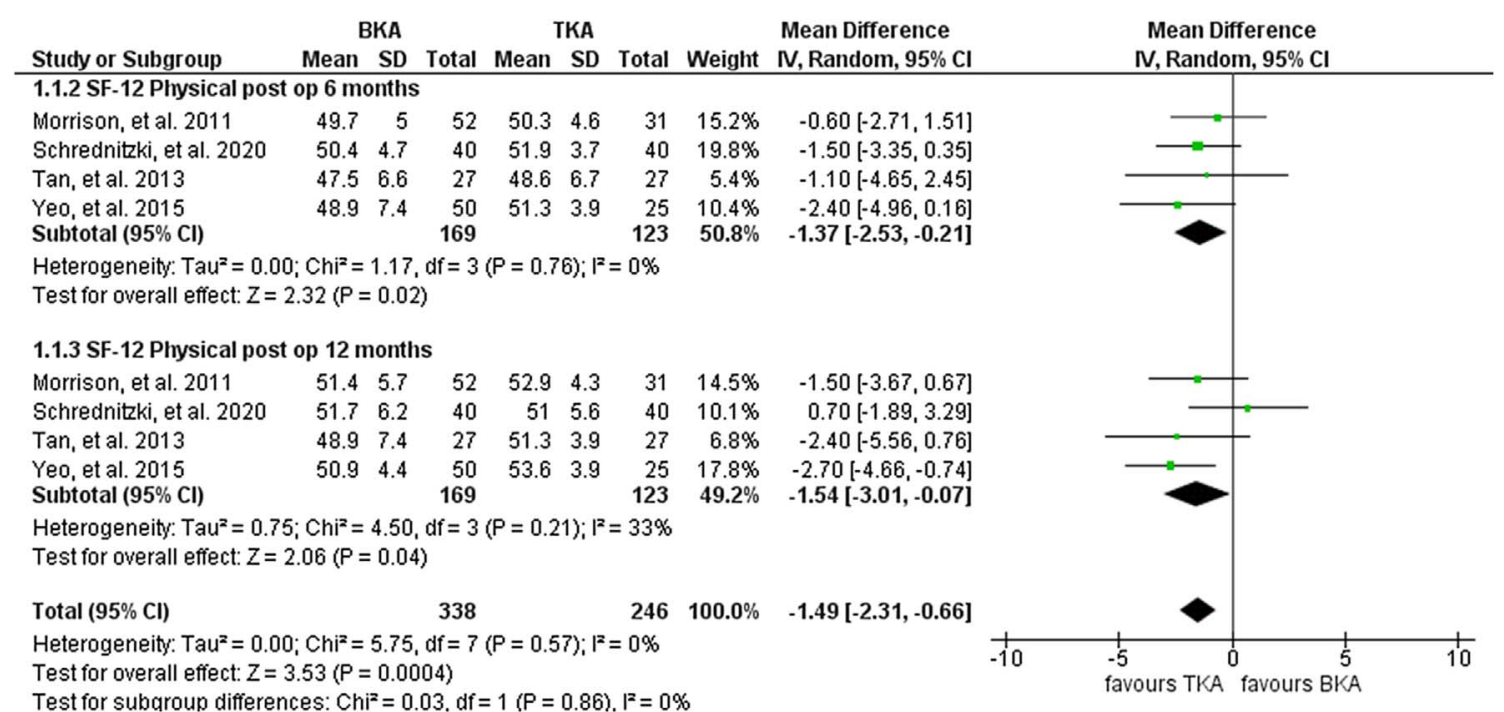

Figure 6. Forest plot of comparison: BKA vs TKA, outcome: SF-12 after 6 and 12 months.

the author also raised concerns regarding the design of the BKA prosthesis and it is technically demanding implantation when used for both unicompartmental and patellofemoral osteoarthritis [25].

Chung and Min [27] compared the quadriceps muscle strength between BKA and TKA patients. They could not detect any significant difference between the two cohorts despite the theoretical advantage for the BKA due to the preservation of cruciate ligaments and greater bone stock. Benazzo et al. [28], reported a revision rate of $10 \%$ (3 out 30) for BKA, two of them were for patella resurfacing and the other one was due to aseptic loosening all within 5 years. This was more than quadruple the revision rate for the TKA $(2.23 \%)$ within the same time frame [29]. Another study reported revision of 2 cases out of $41(4.8 \%)$ for BKA at 6 years follow up, with the main reason for revision being aseptic loosening and knee pain [30]. Theoretically, the BKA mimics the native knee kinematics by preserving the cruciate ligaments and good bone stock [31-36]. However, the progression of Osteo Arthritis (OA) in the third compartment raises a major concern for BKA [26]. After a mean of $11.8( \pm 5.4)$ years follow-up, Heyse et al. [26], reported progression of OA within the third compartment in about $55 \%$ of patients (five out of nine) who had undergone BKA. A long-term study examined both the BKA and The TKA for 10 years follow up and did not report any significant difference between the two cohorts, however, the number of patients included in each group was small (26 and 22 respectively) [15]. Moreover, the high percentage of loss to follow up ( $15 \%$ and $22.7 \%$ respectively) means we must take these results with caution. The marginal advantage in the postoperative ROM for the BKA does not make up for the complexity of the surgical technique and the higher revision rate and inferior PROMS reported for it. Moreover, the less intraoperative bleeding for BKA is outweighed by the shorter operative time reported for the TKA. Overall, the BKA did not show any significant advantages over the TKA in the context of $\mathrm{OA}$ and thus we recommend against it.

\section{Study strengths and limitations}

One of the strengths of our study is the large number of studies included in our analysis (11 studies). As well as this a significant portion of our included studies is modern, with all included studies being published between 2009 and 2020. In terms of study limitations, the data used in this study was obtained from several studies reporting the ROM and PROMs between the BKA and TKA. The techniques and materials used in these studies were similar but not identical. Another limitation is the inclusion of five retrospective studies in the meta-analysis. The observational patterns associated with retrospective cohort studies are more susceptible to bias in data collection. Another source of limitation was the lack of longterm follow-up. While the studies included reported scores for up to 5 years follow-up, there is a paucity of data beyond this. We would recommend more RCTs with a long-term follow-up period examining these two prosthetic designs.

\section{Conclusion}

In terms of KSS, OKS, and SF-12, this meta-analysis suggests better short-term results for the TKA compared with the BKA. The TKA was also associated with a shorter operative time and a revision rate similar to BKA at short-term follow-up. The BKA implant showed marginally lower intraoperative blood loss and slightly better ROM. However, it also reported a relatively high failure rate in mid and long-term follow-up in comparison to the TKA that led us to advise against its use.

\section{Contributors}

All authors contributed to the study's conception and design. Conceptualization: [Hany Elbardesy and Ahmed K. Awad], Methodology: [Shane Guerin and Somaya 
Zain Elabdeen Sayed], Formal analysis and investigation: [Hany Elbardesy and Ahmed K. Awad], Writing - original draft preparation: [Hany Elbardesy, Ahmed K. Awad, Samar Tarek Farahat, and André McLeod]; Writing - review and editing: [Hany Elbardesy and James Harty], Supervision: [James Harty and Shane Guerin] and all authors commented on previous versions of the manuscript. All authors read and approved the final manuscript.

\section{Compliance with ethical standards}

Conflict of Interest: The authors declare that they have no conflict of interest.

Funding: There is no funding source.

Ethical approval: This article does not contain any studies with human participants or animals performed by any of the authors.

Informed consent: not applicable.

\section{References}

1. AOA (2020) Annual 2020. Aust Orthop Assoc Natl Jt Replace Regist 21, 219-289.

2. Evans JT, Walker RW, Evans JP, et al. (2019) How long does a knee replacement last? A systematic review and meta-analysis of case series and national registry reports with more than 15 years of follow-up. Lancet 393, 655-663

3. Wang W, Sun M, Palmer J, et al. (2018) Patterns of compartment involvement in end-stage knee osteoarthritis in a Chinese Orthopedic Center: Implications for implant choice. Orthop Surg 10, 227-234.

4. Heyse TJ, El-Zayat BF, De Corte R, et al. (2014) UKA closely preserves natural knee kinematics in vitro. Sport Traumatol Arthrosc 22, 1902-1910.

5. Heyse TJ, Slane J, Peersman G, et al. (2017) Kinematics of a bicruciate-retaining total knee arthroplasty. Knee Surg Sport Traumatol Arthrosc 25, 1784-1791.

6. Siman H, Kamath AF, Carrillo N, et al. (2017) Unicompartmental knee arthroplasty vs total knee arthroplasty for medial compartment arthritis in patients older than 75 Years: Comparable reoperation, revision, and complication rates. J Arthroplasty 32, 1792-1797.

7. Deng M, Hu Y, Zhang Z, et al. (2021) Unicondylar knee replacement versus total knee replacement for the treatment of medial knee osteoarthritis: a systematic review and metaanalysis. Arch Orthop Trauma Surg. https://doi.org/10.1007/ s00402-021-03790-7.

8. Kennedy JA, Mohammad HR, Mellon SJ, et al. (2020) Age stratified, matched comparison of unicompartmental and total knee replacement. Knee 27, 1332-1342.

9. Higgins JPT, Thomas J, Chandler J, Cumpston M, Li T, Welch VA, Page MJ (2019) Cochrane Handbook for Systematic Reviews of Interventions.

10. Wells GA, Shea B, O'Connell D, Peterson J, Welch VLM The NewcastleeOttawa Scale (NOS) for Assessing the Quality of Non-randomized Studies in Meta-analysis. Ottawa Heal Res Inst 1, 1-12. (Accessed 10.01.2021)
11. Morrison TA, Nyce JD, Macaulay WB, Geller JA (2011) Early adverse results with bicompartmental knee arthroplasty: A prospective cohort comparison to total knee arthroplasty. J Arthroplasty 26, 35-39.

12. Yeo NEM, Chen JY, Yew A, et al. (2015) Prospective randomised trial comparing unlinked, modular bicompartmental knee arthroplasty and total knee arthroplasty: A five years follow-up. Knee 22, 321-327.

13. Schrednitzki D, Beier A, Marx A, Halder AM (2020) No major functional benefit after bicompartmental knee arthroplasty compared to total knee arthroplasty at 5-Year follow-up. J Arthroplasty 35, 3587-3593.

14. Engh GA, Parks NL, Whitney CE (2014) A prospective randomized study of bicompartmental vs. total knee arthroplasty with functional testing and short term outcome. J Arthroplasty 29, 1790-1794.

15. Goh JKM, Chen JY, Yeo NEM, et al. (2020) Ten year outcomes for the prospective randomised trial comparing unlinked, modular bicompartmental knee arthroplasty and total knee arthroplasty. Knee 27, 1914-1922.

16. Parratte S, Ollivier M, Opsomer G, et al. (2015) Is knee function better with contemporary modular bicompartmental arthroplasty compared to total knee arthroplasty? Short-term outcomes of a prospective matched study including 68 cases. Orthop Traumatol Surg Res 101, 547-552.

17. Ko Y, Lo N-N, Yeo S-J, et al. (2013) Comparison of the responsiveness of the SF-36, the Oxford Knee Score, and the Knee Society Clinical Rating System in patients undergoing total knee replacement. Qual Life Res 22, 2455-2459.

18. Giesinger JM, Hamilton DF, Jost B, et al. (2015) WOMAC, EQ-5D and knee society score thresholds for treatment success after total knee arthroplasty. J Arthroplasty 30, 2154-2158.

19. Lin Y, Yu Y, Zeng J, et al. (2020) Comparing the reliability and validity of the SF-36 and SF-12 in measuring quality of life among adolescents in China: a large sample cross-sectional study. Health Qual Life Outcomes 18, 360.

20. CTNC, Collaboration (2014) RM (RevMan) [Computer P, Centre V 5. 3]. TC Review Manager vol 5.0.

21. Siddharth S, Dutton A, Liang S, Dasde S (2013) Bicompartmental versus total knee arthroplasty for medio-patellofemoral osteoarthritis: A comparison of early clinical and functional outcomes. J Knee Surg 26, 411-416.

22. Tan SM, Dutton AQ, Bea KC, Kumar VP (2013) Bicompartmental versus total knee arthroplasty for medial and patellofemoral osteoarthritis. J Orthop Surg (Hong Kong) 21, 281-284.

23. Biazzo A, Silvestrini F, Manzotti A, Confalonieri N (2019) Bicompartmental (uni plus patellofemoral) versus total knee arthroplasty: a match-paired study. Musculoskelet Surg 103, $63-68$.

24. Ma JX, He WW, Kuang MJ, et al. (2017) Efficacy of bicompartmental knee arthroplasty (BKA) for bicompartmental knee osteoarthritis: A meta analysis. Int J Surg 46, 53-60.

25. Parratte S, Pauly V, Aubaniac J-M, Argenson J-NA (2010) Survival of bicompartmental knee arthroplasty at 5 to 23 Years. Clin Orthop Relat Res 468(1), 64-72.

26. Heyse TJ, Khefacha A, Cartier P (2010) UKA in combination with PFR at average 12-year follow-up. Arch Orthop Trauma Surg 130, 1227-1230.

27. Chung JY, Min B-H (2013) Is bicompartmental knee arthroplasty more favourable to knee muscle strength and physical performance compared to total knee arthroplasty? Knee Surg Sport Traumatol Arthrosc 21, 2532-2541. 
28. Benazzo F, Rossi SMP, Ghiara M (2014) Partial knee arthroplasty: patellofemoral arthroplasty and combined unicompartmental and patellofemoral arthroplasty implants - general considerations and indications, technique and clinical experience. Knee 21, S43-S46.

29. Brittain R, Dawson-bowling S, Goldberg A, et al. (2020) 17th Annual Report. Wales, North Irel Isle Man, Natl Jt Regist England, 120-193.

30. Romagnoli S, Marullo M (2018) Mid-Term clinical, functional, and radiographic outcomes of 105 gender-specific patellofemoral arthroplasties, with or without the association of medial unicompartmental knee arthroplasty. J Arthroplasty 33, 688-695.

31. Banks SA, Fregly BJ, Boniforti F, et al. (2005) Comparing in vivo kinematics of unicondylar and bi-unicondylar knee replacements. Knee Surg Sport Traumatol Arthrosc 13, 551-556.
32. Confalonieri N, Manzotti A, Cerveri P, De Momi E (2009) Bi-unicompartmental versus total knee arthroplasty: a matched paired study with early clinical results. Arch Orthop Trauma Surg 129, 1157-1163.

33. Fuchs S, Tibesku CO, Frisse D, et al. (2005) Clinical and functional comparison of uni- and bicondylar sledge prostheses. Knee Surg Sport Traumatol Arthrosc 13, 197-202.

34. Fuchs S, Tibesku CO, Genkinger M, et al. (2003) Proprioception with bicondylar sledge prostheses retaining cruciate ligaments. Clin Orthop Relat Res 406, 148-154.

35. Rolston L, Bresch J, Engh G, et al. (2007) Bicompartmental knee arthroplasty: a bone-sparing, ligament-sparing, and minimally invasive alternative for active patients. Orthopedics 30, 70-3.

36. Sabatini L, Giachino M, Risitano S, Atzori F (2016) Bicompartmental knee arthroplasty. Ann Transl Med 4, 1-7.

Cite this article as: Elbardesy H, Awad AK, McLeod A, Farahat ST, Sayed SZE, Guerin S \& Harty J (2021) Does bicompartmental knee arthroplasty hold an advantage over total knee arthroplasty? Systematic review and meta-analysis. SICOT-J 7, 38 